\title{
L'intervention EMDR rapide après un incident critique dans une collectivité : un essai clinique randomisé
}

\author{
Elan Shapiro \\ Haifa, Israël \\ Brurit Laub \\ Rehovot, Israël
}

\begin{abstract}
Le but de cette étude était d'investiguer l'efficacité de l'intervention EMDR (désensibilisation et retraitement par les mouvements oculaires) rapide à l'aide du protocole EMDR de l'épisode traumatique récent (EMDR recent traumatic episode protocol [R-TEP]) après un événement traumatique dans une collectivité, lors duquel un missile a frappé un bâtiment dans un quartier très fréquenté de la ville. Dans un essai contrôlé randomisé de groupes parallèles avec liste d'attente/traitement reporté, 17 rescapés manifestant une détresse post-traumatique ont été traités avec la psychothérapie EMDR en utilisant le protocole R-TEP. Des praticiens EMDR bénévoles ont effectué le traitement lors de deux journées consécutives. Les participants étaient assignés de manière aléatoire à la condition du traitement immédiat ou à celle de la liste d'attente/traitement reporté. Les évaluations à l'aide de l'échelle Impact of Event ScaleRevised (Échelle révisée d'impact de l'événement [IES-R]) et de l'inventaire bref de la dépression du Patient Health Questionnaire (PHQ-9) ont été réalisées lors du pré- et du post-traitement ainsi qu'au suivi après trois mois. Une semaine post-traitement, les scores du groupe de traitement immédiat s'étaient significativement améliorés sur l'IES-R en comparaison du groupe liste d'attente/traitement reporté qui n'a montré aucune amélioration avant le traitement. Lors du suivi après trois mois, les résultats de l'IES$\mathrm{R}$ étaient maintenus et les scores PHQ-9 montraient une amélioration significative. Cette étude pilote apporte des preuves préliminaires qui soutiennent l'efficacité de l'EMDR R-TEP dans la réduction du stress post-traumatique chez les victimes civiles d'hostilités et qui montrent que ce modèle d'intervention, brièvement ajouté aux services locaux à la suite d'incidents traumatiques à grande échelle, fondé sur une intervention EMDR sur deux jours consécutifs, peut être efficace.
\end{abstract}

Mots-clés : EMDR ; intervention EMDR rapide ; protocole de l'épisode traumatique récent (R-TEP); stress post-traumatique en santé mentale des catastrophes ; dépression post-traumatique

À

huit heures du matin, le 14 novembre 2012, un missile de Gaza a frappé un bâtiment dans une communauté religieuse dans le Sud d'Israël. Trois individus furent tués : un homme de vingt ans, une mère de jeunes enfants (son fils fut également blessé) et un père d'enfants en bas âge. Les immeubles de ce quartier sont surpeuplés et la communauté était fortement ébranlée, d'autant plus qu'elle possède la croyance religieuse profondément ancrée qu'elle est protégée par Dieu. Après l'attaque, le psychologue local nota des niveaux d'anxiété élevés au sein de la communauté et demanda de l'aide à l'association EMDR-Israël.

\section{Interventions psychologiques rapides après des événements traumatiques}

Les revues Cochrane des études contrôlées sur les interventions psychologiques rapides après des événements traumatiques (Bisson \& Andrew, 2007 ; Roberts, Kitchiner, Kenardy \& Bisson, 2008, 2009, 2010) ont défini l'intervention psychologique rapide comme

This article originally appeared as Shapiro, E. \& Laub, B. (2015). Early EMDR Intervention Following a Community Critical Incident: A Randomized Clinical Trial. Journal of EMDR Practice and Research, 9(1), 17-27. Translated by Jenny Ann Rydberg. 
commençant dans les trois mois suivant l'événement traumatique, dans un but de prévention ou de traitement de l'état de stress post-traumatique (ESPT) ou de la détresse continue, de l'état de stress aigu ou d'autres troubles associés au trauma. La dernière revue Cochrane, publiée en 2010, contenait des articles publiés jusqu'en juillet 2008. Quinze études étaient identifiées, comprenant des essais contrôlés randomisés sur toute intervention ou tout traitement psychologiques destinés à réduire les symptômes de stress traumatique aigu, à l'exception des interventions à séance unique. Il n'y avait pas d'étude éligible sur l'EMDR. Les interventions brèves de thérapie cognitive comportementale centrée sur le trauma (TCC-CT) étaient plus efficaces qu'une intervention liste d'attente ou des entretiens de soutien. Cependant, des réserves ont été émises quant à la qualité des essais et les auteurs ont formulé la recommandation selon laquelle « des essais de grande qualité supplémentaires avec des périodes de suivi plus longues sont nécessaires pour tester plus loin la TCC-CT et d'autres formes d'intervention psychologique " (Roberts et coll., 2010, p. 2).

La plupart des personnes exposées à un trauma connaîtront des symptômes transitoires de stress traumatique. Toutefois, $45 \%$ à $80 \%$ se seront naturellement rétablis après neuf mois, en fonction du type d'exposition et d'autres variables personnelles (National Institute for Clinical Excellence [NICE], 2005). On peut donc arguer qu'une intervention rapide n'est pas nécessaire et que nous pourrions simplement attendre de voir qui présente encore des symptômes et ne traiter que ces individus-là. D’un autre côté, 33\% restent symptomatiques pendant trois ans ou plus, avec un risque accru de problèmes secondaires. Cela reste un nombre important de personnes. Même des symptômes infracliniques résiduels et des complications secondaires peuvent avoir un impact étendu sur la qualité de vie et les relations.

Le stress traumatique est un facteur de risque pour de nombreuses plaintes psychologiques et somatiques. Même lorsque seuls des symptômes infracliniques sont présents, l'ESPT ou d'autres troubles associés peuvent connaître une apparition différée, se développant plusieurs mois ou années plus tard ou suite à une exposition subséquente à un trauma (Andrews, Brewin, Philpott \& Stewart, 2007 ; Bryant, Creamer, O’Donnell, Silove \& McFarlane, 2011 ; McFarlane, 2009, 2010a, 2010b). La plupart des victimes du trauma présentant des symptômes ne chercheront pas de traitement (Brewin et coll., 2010) donc l'apport d'une aide assez rapide pourrait réduire l'évitement d'un traitement. L'intervention EMDR comporte plusieurs avantages : elle est brève, ne requiert pas de tâche à réaliser en dehors des séances et peut s'effectuer lors de journées consécutives. L'intervention EMDR rapide (IER) pourrait également jouer un rôle préventif en réduisant l'accumulation de souvenirs traumatiques stockés de manière dysfonctionnelle et en renforçant les liens avec des réseaux mnésiques adaptatifs, favorisant ainsi l'intégration des expériences traumatiques et augmentant la résilience.

\section{Intervention EMDR rapide}

La psychothérapie EMDR est un traitement de l'ESPT fondé sur les preuves. Plus de 24 études contrôlées randomisées ont été réalisées sur l'efficacité de l'EMDR dans le traitement du trauma. La psychothérapie EMDR est approuvée en tant que l'un des traitements de choix de l'ESPT par de nombreuses organisations nationales et internationales de santé mentale, incluant l'Organisation mondiale de la santé (WHO, 2013), NICE (2005), la Société américaine de psychiatrie (APA, 2004) et le Conseil national israélien pour la santé mentale (voir Bleich, Kotler, Kutz \& Shalev, 2002).

Plusieurs protocoles IER sont décrits dans la littérature (voir Luber, 2013 ; EMDR Research Foundation). Francine Shapiro $(1995,2001)$ proposa le protocole originel de l'événement traumatique récent sous forme d'une application du protocole EMDR standard, concevant l'événement traumatique comme une expérience fragmentée non encore consolidée de telle sorte qu'une seule image ne puisse pas représenter l'événement entier, nécessitant ainsi un protocole EMDR différent, multi-cibles. Après la préparation, le patient est invité à raconter l'histoire de l'événement traumatique en notant les segments de perturbation et en en identifiant le plus perturbant (la première cible). Le segment le plus perturbant est évalué et traité comme dans le protocole EMDR standard, jusqu'à ce que le SUD (unités subjectives de perturbation) atteigne un niveau écologique et que la cognition soit installée. Ces étapes sont répétées par ordre chronologique avec d'autres segments traumatiques de l'événement. Quand le patient peut visualiser l'événement depuis le début jusqu'à la fin sans détresse (à moins qu'elle soit écologique), il est invité à développer une cognition positive $(\mathrm{CP})$ pour l'événement entier qu'il visualise avec la $\mathrm{CP}$, les yeux ouverts, pendant les stimulations bilatérales. Puis le scanner corporel et la clôture sont effectués et, lors de la réévaluation, les déclencheurs sont retraités si nécessaire et un scénario futur est réalisé pour chaque déclencheur présent.

Plus tard, Shapiro a réintroduit son protocole EMD originel pour les situations d'urgence (F. Shapiro, 
2004). Le protocole EMD diffère du protocole EMDR standard en ce qu'il se centre sur l'image cible et y revient régulièrement en vérifiant le SUD, limitant ainsi les chaînes associatives. Une autre version modifiée sur protocole de l'événement récent (1995, 2001), appelé le protocole EMDR pour les incidents critiques récents (EMDR-PRECI), fut récemment présenté (Jarero, Artigas \& Luber, 2011 ; Jarero \& Uribe, 2011). Ce protocole comporte également quelques similitudes avec le protocole de l'événement récent ainsi qu'avec le protocole EMDR de l'épisode traumatique récent $(\mathrm{R}-\mathrm{TEP})$. Après la préparation, le patient est invité à faire défiler le film de l'événement, commençant juste avant le début, jusqu'à aujourd'hui. La première cible sélectionnée est la plus perturbante et les autres sont identifiées en demandant au patient de visualiser à nouveau toute la séquence de l'événement, les yeux fermés. Les cibles sont évaluées sans la CP et le VOC, puis traitées comme dans le protocole EMDR standard. Si aucune perturbation n'apparaît pendant la visualisation de l'événement entier, les yeux ouverts, la CP pour tout l'événement est identifiée et installée avec les stimulations bilatérales du câlin $\mathrm{du}$ papillon. Jarero et Uribe (2012) suggèrent qu'une séance d'EMDR-PRECI suffit à résoudre les expériences traumatiques en cours.

\section{Le protocole EMDR de l'épisode traumatique récent}

L'EMDR R-TEP (E. Shapiro \& Laub, 2008, 2014 ; E. Shapiro, 2009, 2012) est un protocole structuré, intégratif et complet, centré sur le trauma récent pour l'IER. Il comprend une adaptation des protocoles EMD et de l'événement récent ainsi que d'autres procédures spécifiques et mesures additionnelles pour la contenance et la sécurité. Les interventions aiguës concernent généralement des personnes normales qui ont été exposées à des situations anormales. L'EMDR $\mathrm{R}$-TEP comporte donc des recommandations proposant un contrat thérapeutique initial qui applique des stratégies d'intervention minimales centrées sur le trauma actuel. Il propose un contrôle de la santé mentale, non seulement dans le but de traiter la détresse traumatique mais aussi pour prévenir les complications post-traumatiques et l'accumulation de souvenirs traumatiques. L'EMDR R-TEP est une intervention brève offrant potentiellement des effets thérapeutiques rapides, généralement en deux à quatre séances. Au moins deux séances sont requises car le suivi est considéré comme essentiel. L'intervention peut se dérouler lors de journées consécutives car aucune tâche ne doit être réalisée entre les séances, ce qui présente un avantage en cas de détresse importante mais également du point de vue des équipes sur le terrain.

L’incidentcritiqueinitial,combinéàsesconséquences traumatiques, est considéré comme un continuum constitué d'un épisode traumatique continu car les expériences ne sont pas encore consolidées ni n'ont été traitées de manière adaptative. L'épisode comporte divers événements traumatiques depuis l'incident initial jusqu'à aujourd'hui, avec de multiples cibles de perturbation. Ces fragments-cibles sont appelés points de perturbation (points of disturbance [PoDs]). Après un premier dépistage et l'enseignement d'exercices d'autostabilisation, le patient est invité à raconter à voix haute l'histoire traumatique de l'épisode, tout en recevant des stimulations bilatérales incluant de préférence des mouvements oculaires pour le maintien de la conscience double et l'ancrage dans la sécurité présente (E. Shapiro, 2011). Il s'agit d'un traitement initial qui permet de combler certains des trous de l'histoire traumatique. La procédure suivante est la « recherche Google » qui équivaut à un balayage interne non séquentiel de l'épisode en vue d'identifier un PoD. Ce fragment-cible est évalué, comme dans le protocole EMDR standard, puis retraité à l'aide de stratégies qui limitent les associations aux frontières de l'épisode traumatique. Toute association qui sort de l'épisode est accueillie, mais le patient est alors invité à revenir à la cible pour se recentrer et vérifier le SUD. Cette délimitation permet un retraitement centré sur l'épisode, qui suffit généralement à une résolution adaptative de l'épisode. Si le patient n'atteint pas une résolution adaptative, on peut lui offrir, avec son consentement, un travail à l'aide du protocole EMDR standard pour un retraitement plus étendu. Le traitement optimal des fragments intrusifs a lieu avec la stratégie EMD qui comprend une focalisation associative étroite sur le seul fragment-cible. Il s'agit le plus souvent d'une procédure brève qui peut être particulièrement efficace dans la réduction rapide de la perturbation des fragments intrusifs (Kutz, Resnik \& Dekel, 2008). Si le SUD de la cible PoD est réduite à un niveau écologique (réaliste), alors le thérapeute installera une CP. Quand la recherche Google ne révèle plus de fragment-cible, le thérapeute passe au niveau de l'épisode pour terminer. On vérifie le SUD de l'épisode entier et s'il est écologique, on détermine la CP de tout l'épisode et on l'installe. Puis on réalise le scanner corporel pour la première fois et le traitement se termine par des procédures de stabilisation. Chaque séance au cours de la thérapie se termine avec une clôture forte et contenante, se centrant sur les ressources.

Le protocole EMDR R-TEP est guidé par une conceptualisation théorique de la nature du processus 
de consolidation de la mémoire du système de traitement adaptatif de l'information après un trauma récent (Laub \& Weiner, 2011 ; E. Shapiro \& Laub, 2008, 2009, 2014 ; Tofani \& Wheeler, 2011). Un élargissement progressif du traitement est suggéré depuis le fragment vers l'événement, l'épisode, jusqu'au thème et à l'identité. Les fragments sensorimoteurs sont généralement prédominants au début et la stratégie EMD, qui délimite de manière étroite et focalisée les associations au seul fragment-cible (PoD) intrusif, favorise un traitement bref et contenu correspondant à cette étape. Plus tard, des expériences plus complexes des événements traumatiques, contenant des informations émotionnelles plus marquantes, émergent en vue du retraitement. La stratégie EMDr, avec une délimitation plus large des associations en lien avec l'épisode traumatique actuel, est appropriée ici, maintenant un retraitement contenu, focalisé sur l'épisode. Ceci peut servir comme un pont vers la focalisation étendue du protocole EMDR standard avec ses associations libres illimitées et une organisation cognitive croissante. La résolution adaptative de l'épisode favorise des liens adaptatifs vers des réseaux mnésiques traumatiques passés et vers des réseaux futurs, produisant une affirmation de soi, un coping et une résilience.

\section{Études évaluant des interventions EMDR rapides}

Bien que de nombreuses questions demeurent concernant l'applicabilité et l'efficacité de la thérapie du trauma dans des contextes post-catastrophe et malgré les difficultés au niveau de la réalisation de recherches dans ces circonstances, il existe un corpus croissant de preuves de l'utilisation efficace de l'EMDR dans le traitement de l'ESPT après des catastrophes d'origine naturelle ou humaine (Buydens, Wilensky \& Hensley, 2014 ; Colelli \& Patterson, 2008 ; Gelbach, 2008 ; Grainger, Levin, AllenByrd, Doctor \& Lee, 1997 ; Maxfield, 2008 ; Natha \& Daiches, 2014 ; E. Shapiro, 2012 ; E. Shapiro \& Laub, 2014 ; Silver, Rogers, Knipe \& Colelli, 2005).

Dans leur revue des interventions EMDR après des catastrophes naturelles, Natha et Daiches (2014) ont identifié huit études faisant état de preuves de l'efficacité réelle et potentielle de l'EMDR dans le traitement de symptômes d'anxiété, de dépression et d'autres formes de détresse psychologique chez les rescapés de catastrophes naturelles.

Il y avait quatre essais contrôlés, un essai partiellement contrôlé et trois études non contrôlées. Les auteurs ont conclu que toutes les études passées en revue ont démontré une signification statistique et clinique au niveau de la réduction de la détresse psychologique chez les rescapés de catastrophes naturelles. À propos des limites de la méthodologie et des plans de recherche, ils ont noté que la nature des catastrophes rend difficile la mise en ouvre complète des critères de recherches "Gold Standard " révisés (Maxfield \& Hyer, 2002), tels que des assesseurs aveugles, l'utilisation de mesures autres que celles d'autoévaluation et le recueil de l'histoire de problèmes préexistants de santé mentale et de la psychothérapie concomitante.

Toutefois, on constate une pénurie de recherches contrôlées sur l'efficacité des protocoles d'intervention rapide dans le traitement des symptômes post-traumatiques aigus après des catastrophes d'origine humaine. Jarero et Uribe $(2011,2012)$ ont effectué une étude contrôlée avec le protocole EMDR-PRECI auprès de salariés médico-légaux mexicains qui travaillaient avec les corps retrouvés dans des charniers. Ils ont été traités en une seule séance. Pour des raisons éthiques, les groupes n’ont pas été divisés de manière aléatoire. Dix-huit participants, ayant des scores élevés, étaient assignés au groupe expérimental et quatorze, ayant des scores modérés, constituaient le groupe de liste d'attente/traitement différé. Les résultats de pré- et de post-test ainsi que du suivi après trois et cinq mois ont montré une amélioration pour les groupes tant de traitement immédiat que de liste d'attente / traitement différé à l'aide des mesures IES et Short PTSD Rating Interview (Entretien court d'évaluation de l'ESPT).

En plus de cette étude, d'autres études soutenant l'utilisation de l'EMDR R-TEP dans le traitement du trauma aigu ont été présentées lors de congrès et sont en cours de publication. Après les séismes dans le nord de l'Italie à l'été 2012, l'EMDR R-TEP a été employé auprès de plus de mille rescapés. Les résultats ont indiqué que ce protocole EMDR constitue une intervention rapide efficace auprès d'une population affectée par une catastrophe naturelle. En plus de réduire les symptômes d'ESPT, il s'est avéré utile pour des individus n'éprouvant qu'une détresse psychologique et pour ceux qui ne pouvaient être considérés comme relevant d'un ESPT même infraclinique (Fernandez, 2013). Après l'attentat terroriste d'Istanbul en 2009, l'EMDR R-TEP fut utilisé pour traiter les rescapés. En moyenne trois séances de 120 minutes de R-TEP suffisaient pour traiter deux à trois cibles de l'épisode traumatique. Les effets positifs étaient maintenus lors du suivi après six mois (Kaya, 2010). Récemment, deux essais contrôlés randomisés employant les protocoles EMDR R-TEP furent réalisés auprès de réfugiés syriens dans le cadre d'un projet d'envergure intervenant parmi les 225000 individus qui ont fui en traversant la frontière turque 
et qui vivent dans des camps de réfugiés. Au moyen d'essais randomisés, contrôlés par liste d'attente, auprès de 29 participants dans la première étude pilote (15 dans le groupe expérimental et 14 dans le groupe contrôle) et de 59 dans la deuxième étude ( 32 dans le groupe expérimental et 27 dans le groupe contrôle). Les études ont conclu que l'EMDR R-TEP est efficace dans la réduction de l'ESPT et des symptômes dépressifs chez les réfugiés (Yurtsever et coll., 2014).

\section{Buts et hypothèses}

Le but de cette étude était d'investiguer l'efficacité de l'IER en utilisant l'EMDR R-TEP après un événement traumatique dans une collectivité. On formula l'hypothèse selon laquelle on verrait des différences significatives entre le groupe de traitement et le groupe de liste d'attente au niveau des scores post-traumatiques et dépressifs, et qu'il y aurait des différences pré-post significatives au niveau des scores, les effets du groupe de traitement immédiat étant reproduits par le groupe de traitement différé. Un but supplémentaire consistait à tester l'utilisation de ce modèle d'assistance amplifiée brève auprès des ressources locales en santé mentale dans des situations d'urgence, en appliquant un traitement IER lors de deux journées consécutives, avec des psychothérapeutes bénévoles, pour réduire les symptômes de stress post-traumatique et de dépression. L'étude commença six semaines après l'incident. Les psychothérapeutes bénévoles qui participèrent à cette étude provenaient de différentes régions du pays et étaient des praticiens EMDR possédant une formation spécialisée dans l'utilisation de l’EMDR R-TEP.

\section{Méthode}

\section{Participants}

L'échantillon était composé de dix-sept voisins et amis appartenant à la même collectivité, les survivants d'une attaque fatale de missile qui ont demandé un traitement psychologique après l'incident.

\section{Plan}

L'étude a suivi un plan de groupe contrôle par liste d'attente/traitement différé (voir Figure 1).

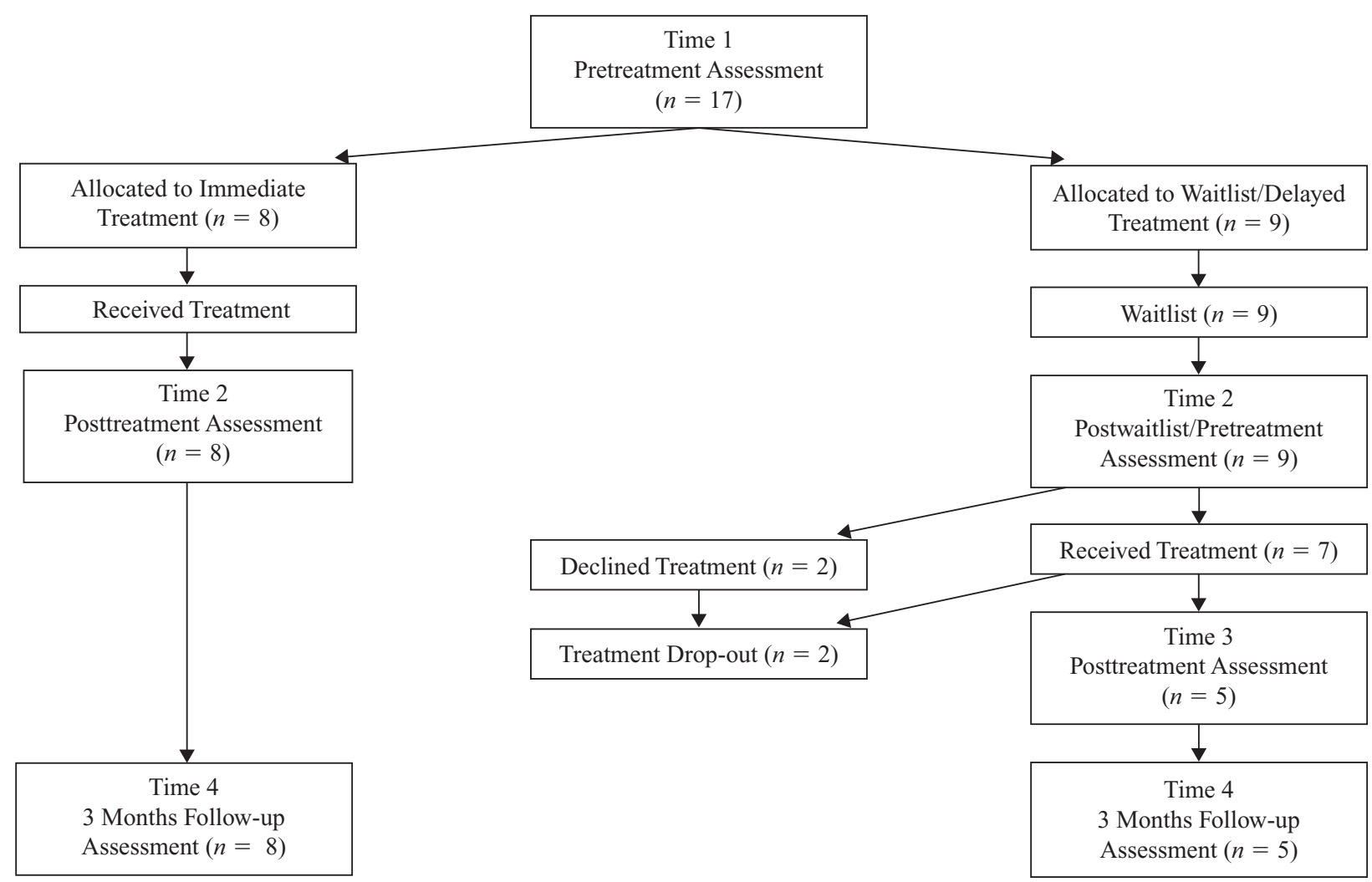

FIGURE 1. Flux des participants à travers l'essai.

Note. Time $=$ Temps ; Pretreatment Assessment $=$ Évaluation pré-traitement ; Allocated to Immediate Treatment $=$ Assigné à un traitement immédiat ; Received Treatment $=\mathrm{A}$ reçu un traitement ; Posttreatment Assessment $=$ Évaluation Post-traitement ; 3 Months Follow-up Assessment = Évaluation de suivi après trois mois ; Allocated to Waitlist/Delayed Treatment $=$ Assigné à la liste d'attente/traitement différé ; Waitlist $=$ Liste d'attente ; Postwaitlist/Pretreatment Assessment $=$ Évaluation post-liste d’attente/pré-traitement. 


\section{Mesures}

Les mesures employées étaient celles de l'échelle révisée d'impact de l'événement (Impact of Event Scale-Revised [IES-R] ; Weiss \& Marmar, 1997) et du questionnaire sur la santé du patient (Patient Health Questionnaire [PHQ-9] ; Spitzer, Kroenke, \& Williams, 1999). L'IES-R est couramment utilisée dans la clinique et dans la recherche en tant qu'échelle d'autoévaluation destinée à mesurer la détresse causée par des événements de vie perturbants. Elle comporte 22 items reflétant les niveaux actuels de détresse, comprenant les symptômes d'activation, de reviviscences et d'évitement. Elle est dérivée des critères du Diagnostic and Statistical Manual of Mental Disorders (4 édition; DSM-IV ; American Psychiatric Association, 1994) pour l'ESPT. Les réponses aux items varient entre 0 (pas du tout) à 4 (extrêmement fort), avec un score maximal de 85 . L'IES-R est une révision de l'IES originelle, reconnue comme l'un des premiers instruments d'autoévaluation développés afin de mesurer le stress post-traumatique, qui ajoute un troisième groupe de symptômes, ceux de l'hyperactivation, à ceux des sous-échelles d'intrusion et d'évitement. Il n’y a pas de valeur seuil spécifique, même si diverses valeurs seuil ont été proposées, de 33 suggéré par Creamer, Bell et Failla (2003) à 22 proposé par Rash, Coffey, Baschnagel, Drobes et Saladind (2008) qui encouragent les chercheurs et les cliniciens à ajuster la valeur seuil sur la base des présentations propres à l'échantillon de la population ciblée.

La PHQ-9 est une échelle brève d'autoévaluation de la dépression qui attribue à chacun des neuf critères DSM-IV un score entre 0 (pas $d u$ tout) et 3 (presque chaque jour), avec un score maximal de 27. Elle est validée pour un usage dans un cadre de soins primaires et possède une validité diagnostique comparable au Primary Care Evaluation of Mental Disorders originel, administré par un clinicien, mais son administration est plus efficiente. La PHQ-9 est un instrument de mesure fiable et valide de la sévérité de la dépression (Kroenke, Spitzer \& Williams, 2001). Elle facilite aussi les diagnostics fondés sur les critères des troubles dépressifs. Des scores de 5, 10, 15 et 20 indiquent une dépression respectivement légère, modérée, moyennement sévère et sévère. Le caractère bref de la PHQ-9 (la passation requiert trois ou quatre minutes) en fait un outil clinique et de recherche particulièrement utile dans l'évaluation des interventions sur le terrain ou lorsque les conditions ne permettent pas d'évaluation plus approfondie. Elle peut même être administrée par téléphone.

\section{Critères d'exclusion}

Tous les participants qui ont demandé un traitement pour des symptômes de détresse ont été évalués ; ils ont obtenu des scores modérés à élevés sur les échelles IES-R et PHQ-9. Trois participants ont été exclus de l'étude en raison d'antécédents de problèmes de santé mentale avant l'attaque ou d'une psychothérapie parallèle.

\section{Procédure}

Les participants de la liste ont été divisés de manière aléatoire en deux groupes : huit participants dans le groupe de traitement immédiat et neuf dans le groupe liste d'attente/traitement différé. Il y avait seize femmes et un homme, âgés de 19 à 57 ans. L'âge moyen était de 42,9 ans ( $\sigma=10,5$ ans) pour le groupe 1 et de 37,1 ans ( $\sigma=14,7$ ans) pour le groupe 2 . Il n'y avait pas de différence d'âge statistiquement significative entre les deux groupes $(t[15]=0,92 ; p=$ 0,37). Les douze praticiens EMDR bénévoles ont été assignés de manière aléatoire au traitement d'un ou de deux participants lors de deux journées consécutives. Les séances duraient environ 90 minutes. Les échelles de mesure étaient administrées par téléphone aux participants par des professionnels aveugles quant au protocole. Tous les participants ont été évalués au temps 1 (T1 ; voir Figure 1). Les huit participants du groupe 1 expérimental étaient invités à bénéficier d'un traitement EMDR R-TEP sur deux journées consécutives. Une semaine plus tard, les participants des deux groupes faisaient l'objet de la même évaluation qu'auparavant (temps 2 [T2]). Le groupe 2 contrôle (liste d'attente/traitement différé) étaient alors invités à bénéficier de leurs séances de traitement. Deux des neuf participants ont refusé le traitement. Ils ont rapporté un bon fonctionnement et leurs scores sur les mesures reflètent une possible rémission spontanée. Deux autres participants ont renoncé à la seconde séance. Les cinq participants restants du groupe 2 étaient évalués sur les deux mesures lors du post-traitement (temps 3 [T3]). Lors du suivi après trois mois, tous les participants qui avaient participé à deux séances ont de nouveau été évalués (temps 4 [T4]).

La fidélité n’a pas été évaluée mais tous les psychothérapeutes étaient des praticiens EMDR formés au protocole R-TEP.

\section{Résultats}

Une analyse de la variance multivariée (MANOVA) a été réalisée afin de comparer les moyennes T1 et T2 des groupes de traitement immédiat et de liste 


\begin{tabular}{|c|c|c|c|c|c|}
\hline & \multirow[b]{2}{*}{ Temps d'évaluation } & \multicolumn{2}{|c|}{$\begin{array}{l}\text { Traitement immédiat } \\
\text { (groupe 1) }\end{array}$} & \multicolumn{2}{|c|}{$\begin{array}{l}\text { Liste d'attente/ traitement différé } \\
\text { (groupe } 2 \text { ) }\end{array}$} \\
\hline & & $\mathrm{m}$ & $\sigma$ & $\mathrm{m}$ & $\sigma$ \\
\hline \multirow[t]{4}{*}{ IES-R } & Temps 1 & 41,63 & 11,46 & 44,28 & 17,54 \\
\hline & Temps 2 & 21,25 & 15,53 & 42,61 & 14,84 \\
\hline & Temps 3 & & & 26,20 & 17,25 \\
\hline & Temps 4 & 15,50 & 12,62 & 24,40 & 14,74 \\
\hline \multirow[t]{4}{*}{ PHQ-9 } & Temps 1 & 13,13 & 3,64 & 10,11 & 5,06 \\
\hline & Temps 2 & 8,88 & 5,41 & 9,44 & 5,66 \\
\hline & Temps 3 & & & 7,20 & 3,96 \\
\hline & Temps 4 & 6,88 & 6,94 & 3,60 & 3,91 \\
\hline
\end{tabular}

Note. IES-R = Impact of Event Scale-Revised ; PHQ-9 = Patient Health Questionnaire ; temps $1=$ prétraitement pour les deux groupes ; temps $2=1$ semaine post-traitement pour le groupe 1 , post-liste d'attente/prétraitement pour le groupe 2 ; temps $3=$ post-traitement pour le groupe 2 ; temps $4=$ suivi après 3 mois pour les deux groupes.

d'attente sur les échelles IES-R et PHQ-9. Pour analyser les résultats de l'étude de reproductibilité, une ANOVA à mesures répétées (employant un modèle mixte) a été réalisée pour l'IES-R et la PHQ-9, comparant les moyennes T1, T2 et T4 du groupe de traitement immédiat avec les moyennes T2, T3 et T4 du groupe de traitement différé. Des tests t post hoc planifiés étaient réalisés lorsque pertinents. Les données ont été analysées à l'aide du SPSS version 21.0. Une valeur de $p$ de 0,05 était considérée statistiquement significative.

\section{Évaluation prétraitement}

Lors de l'évaluation initiale (T1), les scores IES-R moyens des participants en traitement immédiat (groupe $1, \mathrm{~m}$ $=41,63 ; \sigma=11,46)$ et en liste d'attente/traitement différé (groupe $2, \mathrm{~m}=44,28 ; \sigma=17,53$ ) étaient considérés cliniquement significatifs de l'ESPT et ne différaient pas entre eux $(t[15]=0,36 ; p>0,72)$. Les scores PHQ-9 moyens initiaux du groupe $1(\mathrm{~m}=13,13 ; \sigma=3,64)$ et du groupe $2(\mathrm{~m}=10,11 ; \sigma=5,06)$ étaient considérés comme reflétant une dépression modérée (entre 10 et 14) et n'étaient pas significativement différents l'un de l'autre $(t[15]=1,39 ; p>0,18$; voir Tableau 1$)$.

\section{Comparaison du traitement immédiat et de la liste d'attente}

Les tests multivariés sur la différence des scores entre T1 et T2 ont révélé une interaction significative, indiquant une différence significative entre les deux groupes au niveau du changement entre T1 et T2 $(F[2,14]=4,19 ; p<0,04)$. Les tests sur les différences intergroupes n’ont révélé aucun changement pour la PHQ-9 $(F[1,15]=4,19 ; p>0,13)$ mais ont montré une différence statistiquement significative pour l'IES$\mathrm{R}(F[1,15]=8,86 ; p<0,01)$, avec un score pour le groupe de traitement immédiat significativement plus petit lors du post-traitement en comparaison du prétraitement. Une comparaison post-hoc planifiée des scores IES-R au T2 a montré une différence significativement différente entre les deux groupes $(t[15]=$ 2,$98 ; p<0,009$; voir Tableau 1 et Figures 2 et 3).

\section{Comparaison du traitement immédiat et du traitement différé}

L'ANOVA à mesures répétées sur les scores IES-R des groupes de traitement immédiat et de traitement différé a trouvé un effet significatif pour le temps $(F[2,25]=10,68 ; p<0,001)$ mais pas de différence de groupe $(F[1,33]=1,38 ; p>0,25)$ ni d'interaction groupe-temps $(F[2,25]=0,18 ; p>0,84)$. Les tests post hoc ont révélé que l'IES-R était significativement plus faible immédiatement après le traitement $(d=219,23$; erreur-type $=5,88 ; p<0,007)$ et lors du suivi après 3 mois $(d=-23,00$; erreur-type $=5,29 ; p<0,001)$.

L'ANOVA à mesure répétées sur les scores PHQ-9 des groupes à traitement immédiat et différé a trouvé un effet significatif pour le temps $(F[2,25]=5,50 ; p<$ $0,01)$ mais pas de différence de groupe $(F[1,33]=2,60$; $p>0,12)$ ni d'interaction groupe-temps $(F[2,25]=$ $0,09 ; p>0,91)$. Les tests post hoc ont révélé que les scores PHQ-9 était significativement plus faibles lors du suivi après 3 mois en comparaison du prétraitement $(d=-6,38$; erreur-type $=2,03 ; p<0,01)$. 


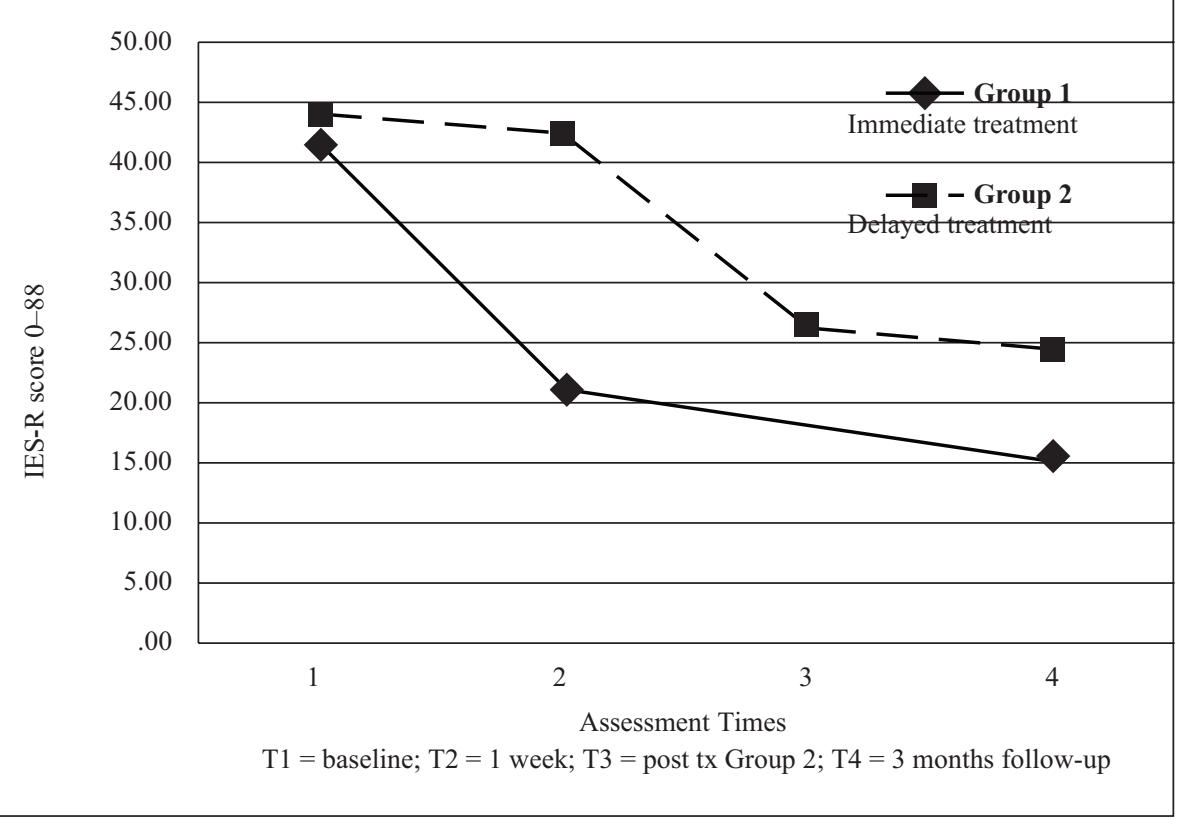

FIGURE 2. Scores moyens à l'IES-R (Impact of Event Scale - Revised) aux quatre temps d'évaluation

Note. Group 1 Immediate treatment = groupe 1 traitement immédiat ; Group 2 Delayed treatment $=$ groupe 2 traitement différé ; Assessment Times = temps d'évaluation ; baseline $=$ ligne de base (évaluation initiale) ; 1 week $=1$ semaine ; posttreatment Group $2=$ post-traitement groupe $2 ; 3$ months follow-up = suivi après 3 mois.

\section{Discussion}

Dans le sillage d'une attaque de missile soudaine et fatale sur une ville, ce projet pilote fut organisé pour apporter assistance à des travailleurs en santé mentale de première ligne qui étaient dépassés. Des bénévoles furent sollicités auprès de l'association EMDR-Israël pour contribuer à l'apport de soins. On leur demanda de mettre en œuvre une intervention brève susceptible d'apporter un soulagement rapide des symptômes de stress post-traumatique, de renforcer la résilience et de prévenir des difficultés supplémentaires en santé mentale. Une intervention sur deux jours consécutifs, employant le protocole EMDR R-TEP, fut choisie car son utilité avait été démontrée auprès d'équipes de bénévoles sur le terrain disposant de ressources et d'un

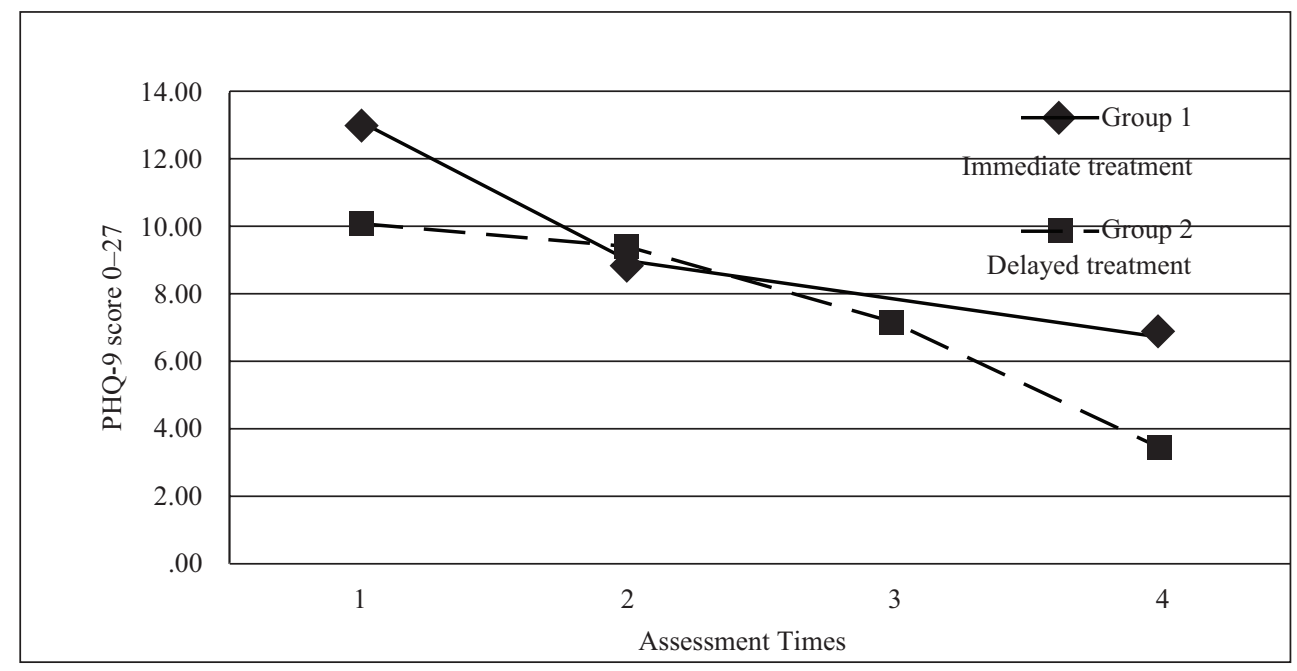

FIGURE 3. Scores moyens de dépression PHQ-9 (Patient Health Questionnaire) au quatre temps d'évaluation

Note. Group 1 Immediate treatment $=$ groupe 1 traitement immédiat ; Group 2 Delayed treatment $=$ groupe 2 traitement différé ; Assessment Times $=$ temps d'évaluation. 
temps limités (Fernandez, 2013). L'étude pilote était conçue de sorte à investiguer l'efficacité de ce modèle d'IER brève.

En plus de la mesure de l'ESPT, une mesure de la dépression fut également employée dans cette étude parce que la dépression est souvent observée à la suite d'un traumatisme, de manière comorbide ou indépendamment de l'ESPT (Bryant, 2011 ; McFarlane, 2010a). Les résultats soutiennent le plus souvent nos hypothèses concernant les mesures de l'ESPT et de la dépression.

Le plan de cette étude employait une comparaison par liste d'attente / traitement différé pour contrôler la rémission spontanée (comparaison par liste d'attente) et pour tenter de répliquer les résultats thérapeutiques (traitement immédiat vs différé). Les scores IES-R du groupe de traitement immédiat se sont significativement améliorés en comparaison du groupe de liste d'attente qui n'a manifesté aucune amélioration avant le traitement. Même si ces résultats suggèrent l'absence d'effet sur les symptômes d'ESPT au niveau de la rémission spontanée pendant cette période d'une semaine, des rémissions spontanées ont pu se produire ultérieurement puisque quatre des neuf participants du groupe de liste d'attente/traitement différé ont refusé ou abandonné le traitement.

La tentative de reproduire les effets thérapeutiques avec le groupe de traitement différé fut compromise par l'attrition dans ce groupe et le faible pouvoir de l'analyse statistique pour les participants restants $(\mathrm{n}=5)$. D’un autre côté, les effets thérapeutiques dans l'étude de réplication ont montré que le traitement résultait en une amélioration significative au cours du temps sur l'IES-R, avec des scores plus faibles lors du post-traitement et du suivi. Cependant, les effets sur la dépression sont plus difficiles à déterminer et auraient pu être plus clairs si une mesure plus sensible de la dépression avait été utilisée (comme l'inventaire de dépression de Beck). Bien qu'il n'y avait pas de différence statistique significative entre les moyennes initiales des groupes sur la mesure de dépression, le score PHQ-9 moyen du groupe contrôle $(10,11)$ était plus proche de la limite entre la dépression faible (5-9) et modérée (10-14), tandis que la moyenne du groupe expérimental $(13,13)$ se situait largement dans la portée de la dépression modérée, ce qui pourrait être cliniquement significatif en ce qui concerne le potentiel de changement après le traitement ou le rétablissement spontané.

\section{Limites}

Notre étude était limitée par le nombre réduit de participants qui diminue la puissance statistique et augmente les effets des différences individuelles au sein du groupe. Par exemple, au sein du groupe de traitement différé, bien que l'âge moyen soit similaire à celui du groupe de traitement immédiat, la variance était bien plus importante (14,7 vs 10,5). Il apparaît qu'il s'agissait des participants les plus jeunes qui soit refusaient soit n'achevaient pas le traitement (âge moyen 34,$8 ; \sigma=15,7)$. D'autres limites comprennent des possibilités limitées quant à la sélection d'admission, le fait de compter sur des mesures d'autoévaluation et la difficulté d'obtenir des vérifications de la fidélité au protocole (les participants religieux refusant d'être filmés). La présomption a été faite qu'ils appartenaient à une communauté solidaire avec des passés culturels et socio-économiques similaires.

\section{Besoin de recherches futures}

La réalisation de recherches sur les interventions rapides qui relèvent de conditions « d'urgence » requiert de la sensibilité et une réponse rapide. Il y a aussi d'inévitables compromis en comparaison d'études réalisées dans des conditions moins stressantes et sans la pression du temps. Il était très utile de disposer d'un modèle de traitement différé comme celui employé par Jarero et al. (2011). Il s'agit d'une manière pragmatique et éthiquement solide de mener des études contrôlées dans de telles circonstances. Dans leurs études, on observa d'importantes améliorations des symptômes de stress post-traumatique tant pour le groupe de traitement immédiat que pour celui de liste d'attente/traitement différé, avec des résultats maintenus lors du suivi, malgré des secousses secondaires continues (Jarero et. al., 2011) et malgré un environnement toujours menaçant (Jarero \& Uribe, 2011, 2012). Dans cette étude, le report d'une semaine $\mathrm{du}$ traitement du groupe contrôle aurait de toute manière été nécessaire pour des raisons logistiques et organisationnelles.

Afin de pouvoir répondre rapidement, de manière efficiente et efficace, des préparations, plans et directives antérieurs sont nécessaires. Cette étude soutient l'appel exprimé par la fondation de recherche EMDR (EMDR Research Foundation) pour l'établissement d'une boîte à outils pour la recherche sur les interventions rapides, comme exprimé dans leur proposition :

La boîte à outils des recherches sur les interventions EMDR rapides a été spécifiquement conçue pour aider les cliniciens EMDR qui apportent des interventions EMDR rapides dans le cadre d'une réponse de première ligne au trauma et du rétablissement. Le premier but de la boîte à outils consiste à augmenter le 
recueil de données et la publication d'études afin d'obtenir une meilleure compréhension des manières les plus efficaces d'intervenir après un événement traumatique. (EMDR Research Foundation, 2014, p. 3)

D’autres études, avec des essais plus importants et des variables supplémentaires, comme le moment de l'intervention, le nombre et l'intensité des séances de traitement, l'exploration de manières efficaces de former rapidement des professionnels de la santé mentale au protocole EMDR R-TEP, et des tests sur d'autres mesures brève de l'ESPT selon les critères DSM-V, de la dépression, ainsi que d'autres mesures de la résilience, sont nécessaires. Un suivi supplémentaire permettrait également de vérifier si les effets thérapeutiques sont maintenus au cours de périodes de temps prolongées et s'ils apportent une protection en termes de résilience dans des situations continues.

\section{Conclusion}

Cette étude pilote apporte des preuves préliminaires suggérant que le protocole EMDR R-TEP est efficace pour réduire le stress post-traumatique et pourrait être utile pour diminuer les symptômes dépressifs chez les victimes civiles d'hostilités. Les résultats suggèrent également ce que modèle rapide et bref de prestation de services en soutien des ressources locales de santé mentale pourrait être efficace après des incidents traumatiques à grande échelle.

\section{Bibliographie}

American Psychiatric Association. (1994). Diagnostic and statistical manual of mental disorders ( $4^{e}$ éd.). Washington, DC: Auteur.

American Psychiatric Association. (2004). Practice guideline for the treatment of patients with acute stress disorder and posttraumatic stress disorder. Arlington, VA: Auteur.

Andrews, B., Brewin, C. R., Philpott, R. \& Stewart, L. (2007). Delayed-onset posttraumatic stress disorder: A systematic review of the evidence. American Journal of Psychiatry, 164(9), 1319-1326.

Bisson, J. \& Andrew, M. (2007). Psychological treatment of post-traumatic stress disorder (PTSD). Cochrane Database of Systematic Reviews, (3), CD003388. http:/ / dx.doi.org/ 10.1002/14651858.CD003388.pub3

Bleich, A., Kotler, M., Kutz, I. \& Shalev, A. (2002). Guidelines for the assessment and professional intervention with terror victims in the hospital and in the community. Paper presented at the National Council for Mental Health, Jerusalem, Israel.

Brewin, C. R., Fuchkan, N., Huntley, Z., Robertson, M., Thompson, M., Scragg, P., . . Ehlers, A. (2010).
Outreach and screening following the 2005 London bombings: Usage and outcomes. Psychological Medicine, 40(12), 2049-2057.

Bryant, R. A. (2011). Acute stress disorder as a predictor of posttraumatic stress disorder: A systematic review. The Journal of Clinical Psychiatry, 72(2), 233-239.

Bryant, R. A., Creamer, M., O’Donnell, M., Silove, D. \& McFarlane, A. C. (2011). The capacity of acute stress disorder to predict posttraumatic psychiatric disorders. Journal of Psychiatric Research, 46(2), 168-173.

Buydens, S., Wilensky, M. \& Hensley, B. J. (2014). Effects of the EMDR protocol for recent traumatic events on acute stress disorder: A case series. Journal of EMDR Practice and Research, 8(1), 2-12.

Colelli, G. \& Patterson, B. (2008). Three case report illustrating the use of the protocol for recent traumatic events following the World Trade Center terrorist attack. Journal of EMDR Practice and Research, 2(2), 114-123.

Creamer, M., Bell, R. \& Failla, S. (2003). Psychometric properties of the Impact of Event Scale-Revised. Behaviour Research and Therapy, 41, 1489-1496.

EMDR Research Foundation. (2014). EMDR early intervention researcher's toolkit. Retrieved from http://emdrresearchfoundation.org/toolkit/toolkit-with-appendices .pdf

Fernandez, I. (2013, June). Early EMDR Intervention (EEI): Theory, practice and research application in a mass disaster. Presented at the EMDR Europe Annual Conference, Geneva, Switzerland.

Gelbach, R. (2008). Trauma, research, and EMDR: A disaster responder's wish list. Journal of EMDR Practice and Research, 2(2), 146-155.

Grainger, R. D., Levin, C., Allen-Byrd, L., Doctor, R. M. $\&$ Lee, H. (1997). An empirical evaluation of eye movement desensitization and reprocessing (EMDR) with survivors of a natural disaster. Journal of Traumatic Stress, 10(4), 665-671.

Jarero, I., Artigas, L. \& Luber, M. (2011). The EMDR protocol for recent critical incidents: Application in a disaster mental health continuum of care context. Journal of EMDR Practice and Research, 5(3), 82-94.

Jarero, I. \& Uribe, S. (2011). The EMDR protocol for recent critical incidents: Brief report of an application in a human massacre situation. Journal of EMDR Practice and Research, 5(4), 156-165.

Jarero, I. \& Uribe, S. (2012). The EMDR protocol for recent critical incidents: Follow-up report of an application in a human massacre situation. Journal of EMDR Practice and Research, 6(2), 50-61.

Kaya, F. (2010, June). The effects of early EMDR interventions (EMD and R-TEP) on the victims of a terrorist bombing in Istanbul. Paper presented at the annual conference of the EMDR Europe Association, Hamburg, Germany.

Kroenke, K, Spitzer R. L. \& Williams J. B. (2001). The PHQ9: Validity of a brief depression severity measure. Journal of General Internal Medicine, 16(9), 606-613. 